\title{
Electron Acceleration by Relativistic Surface Plasmons in Laser-Grating Interaction
}

\author{
L. Fedeli, ${ }^{1,2, *}$ A. Sgattoni, ${ }^{2}$ G. Cantono,,${ }^{3,4,2}$ D. Garzella, ${ }^{3}$ F. Réau, ${ }^{3}$ I. Prencipe,${ }^{5, \dagger}$ M. Passoni, ${ }^{5}$ \\ M. Raynaud, ${ }^{6}$ M. Květoň ${ }^{7}$ J. Proska, ${ }^{7}$ A. Macchi, ${ }^{2,1}$ and T. Ceccotti ${ }^{3}$ \\ ${ }^{1}$ Enrico Fermi Department of Physics, University of Pisa, 56127 Pisa, Italy \\ ${ }^{2}$ National Institute of Optics, National Research Council (CNR/INO), u.o.s Adriano Gozzini, 56124 Pisa, Italy \\ ${ }^{3}$ LIDYL, CEA, CNRS, Université Paris-Saclay, CEA Saclay, 91191 Gif-sur-Yvette, France \\ ${ }^{4}$ University of Paris Sud, Orsay 91405, France \\ ${ }^{5}$ Department of Energy, Politecnico di Milano, Milan 20156, Italy \\ ${ }^{6}$ Laboratoire des Solides irradiés, Ecole Polytechnique, CNRS, CEA/DSM/IRAMIS, \\ Université Paris-Saclay, 91128 Palaiseau Cedex, France \\ ${ }^{7}$ FNSPE, Czech Technical University, Prague 11519, Czech Republic
}

(Received 30 June 2015; published 7 January 2016)

\begin{abstract}
The generation of energetic electron bunches by the interaction of a short, ultraintense $\left(I>10^{19} \mathrm{~W} / \mathrm{cm}^{2}\right)$ laser pulse with "grating" targets has been investigated in a regime of ultrahigh pulse-to-prepulse contrast $\left(10^{12}\right)$. For incidence angles close to the resonant condition for surface plasmon excitation, a strong electron emission was observed within a narrow cone along the target surface, with energy spectra peaking at 5-8 MeV and total charge of $100 \mathrm{pC}$. Both the energy and the number of emitted electrons were strongly enhanced with respect to simple flat targets. The experimental data are closely reproduced by three-dimensional particle-in-cell simulations, which provide evidence for the generation of relativistic surface plasmons and for their role in driving the acceleration process. Besides the possible applications of the scheme as a compact, ultrashort source of $\mathrm{MeV}$ electrons, these results are a step forward in the development of high-field plasmonics.
\end{abstract}

DOI: 10.1103/PhysRevLett.116.015001

Surface plasmons [1,2], also named surface waves, are electromagnetic (EM) modes localized at the interface of different media which allow local field confinement and enhancement. Surface plasmons are the core of the vibrant research field of plasmonics [3], with applications ranging from light concentration beyond the diffraction limit [4], to biosensors [5] and plasmonic chips [6]. The extension of plasmonics into the regime of high fields, where nonlinear and relativistic effects arise, is largely unexplored. An example is provided by the multiterawatt laser-driven excitation of unipolar surface plasmons by transient charge separation $[7,8]$, with potential application to the generation of intense THz pulses [8,9].

In the optical or near-infrared frequency range, surface plasmons can be excited by laser light incident on a sharp material interface having a periodic modulation, e.g., a grating, to allow phase matching. However, most experiments so far have been restricted to intensities below $10^{16} \mathrm{~W} / \mathrm{cm}^{2}$ [10] because of the prepulses inherent in high-power laser systems which can lead to an early disruption of the target structuring. The development of devices for ultrahigh contrast pulses [11,12] now allows us to explore the interaction with targets structured on a submicrometric scale at laser intensities high enough for the electron dynamics to become relativistic $[13,14]$. In particular, a strong increase of the cutoff energy of protons accelerated from the rear surface of grating targets was observed and related to surface plasmon-enhanced absorption [15]. While a detailed theory is still lacking for nonlinear and relativistic surface plasmons, numerical simulations also showed surface plasmon-related effects in this regime [16,17], including electron acceleration at weakly relativistic intensities [18] and, more recently, surface plasmon-enhanced high harmonics [19] and synchrotron radiation [20] in gratings.

In this Letter, we demonstrate that relativistic surface plasmons accelerate high-energy electrons along a grating surface. The acceleration process is related to two basic surface plasmon properties, i.e., the subluminal phase velocity and the longitudinal field component. The energy and number of electrons in gratings irradiated at an incidence angle close to the resonant value for surface plasmon excitation are strongly enhanced with respect to flat targets. At intensities $I=5 \times 10^{19} \mathrm{~W} / \mathrm{cm}^{2}$, corresponding to a relativistic parameter $a_{0} \simeq 5$ [where $a_{0}=\left(I \lambda^{2} / 10^{18} \mathrm{~W} \mathrm{~cm}^{-2} \mu \mathrm{m}^{2}\right)^{1 / 2}$ and $\lambda$ is the laser wavelength] the electron emission was concentrated in a narrow cone with energy spectra peaking at 5-8 $\mathrm{MeV}$ and reaching up to $\sim 20 \mathrm{MeV}$.

The basics of surface plasmon generation and electron acceleration may be described as follows. At high laser intensities $\left(I>10^{18} \mathrm{~W} / \mathrm{cm}^{2}\right)$ a solid target is ionized within one laser cycle, thus the interaction occurs with a dense plasma. Assuming a dielectric function $\varepsilon(\omega)=1-$ $\omega_{p}^{2} / \omega^{2} \equiv 1-\alpha$ (where $\omega_{p}$ is the plasma frequency) the phase velocity of a surface plasmon is $v_{p}=\omega / k=c(\alpha-$ $2)^{1 / 2} /(\alpha-1)^{1 / 2}$ where $k$ is the surface plasmon wave vector 
and $\alpha>2$ holds. The condition for resonant excitation of a surface plasmon on a periodically modulated target (grating) by an incident $\mathrm{EM}$ wave [1,2] of the same frequency is $\lambda / \lambda_{g}=(1-\alpha)^{1 / 2} /(2-\alpha)^{1 / 2}-\sin \left(\phi_{i}\right)$ where $\lambda_{g}$ is the grating period and $\phi_{i}$ is the angle of incidence. Notice that these equations neglect the effects of finite temperature and nonsteplike density profiles [21] as well as of collisions and possible relativistic nonlinearities [22]; thus, in principle the resonance could be expected at somewhat different angles.

Electron acceleration up to relativistic energy by the longitudinal surface plasmon field requires the phase velocity $v_{p}$ to approach $c$ in order to minimize dephasing; thus, $\alpha \gg 1$ is required as expected for a solid-density plasma and optical frequencies. The basic process may be described similarly to the well-known acceleration in wake plasma waves [23], but with the difference that the transverse field component of the surface plasmon (i.e., the field component perpendicular to the wave vector) pushes an electron on the vacuum side, so that the process is two dimensional and eventually the electrons are emitted at an angle with respect to the surface plasmon propagation direction. In a frame $L^{\prime}$ moving with velocity $\mathbf{v}_{p}=v_{p} \hat{\mathbf{y}}$ with respect to the laboratory frame $L$, the surface plasmon field is electrostatic in the vacuum region $(x>0)$ and can be derived from the potential

$$
\Phi^{\prime}=-\frac{E_{\mathrm{SP}}}{k^{\prime}} \mathrm{e}^{-k^{\prime} x} \sin k^{\prime} y^{\prime},
$$

where $k^{\prime}=k / \gamma_{p}, \gamma_{p}=\left(1-v_{p}^{2} / c^{2}\right)^{-1 / 2}=(\alpha-1)^{1 / 2}$, and $E_{\mathrm{SP}}$ is the amplitude of the longitudinal surface plasmon field $\left(E_{y}\right)$ in $L$. In the $L^{\prime}$ frame the process is simply described as the electron going down the potential energy hill $-e \Phi^{\prime}$. Because the evanescent field component $E_{x}^{\prime}=-\partial_{x} \Phi^{\prime}$, electrons are predominantly accelerated towards the vacuum side with velocity almost normal to the $x=0$ surface. The condition of "optimal" injection corresponds to an electron placed initially in $L^{\prime}$ at the top of the potential hill $\left(x^{\prime}=0, y^{\prime}=\pi / 2 k^{\prime}\right)$ with $v_{y}^{\prime}=0$, i.e., with an initial velocity $\mathbf{v}_{p}$ in $L$. Such an electron will acquire in $L^{\prime}$ the energy $W^{\prime}=e E_{\mathrm{SP}} \gamma_{p} / k$. If $a_{\mathrm{SP}} \equiv e E_{\mathrm{SP}} / m_{e} \omega c \sim 1$ then $W^{\prime} \gg m_{e} c^{2}$. In this limit, the energy momentum in $L^{\prime}$ is $p_{\mu}^{\prime} \simeq\left(W^{\prime}, W^{\prime} / c, 0,0\right)$ and thus $p_{\mu} \simeq$ $\left(\gamma_{p} W^{\prime}, W^{\prime} / c, \gamma_{p} W^{\prime} / c, 0\right)$ in $L$. The final energy $\mathcal{E}_{f}$ and emission angle $\phi_{e}$ are given by

$\mathcal{E}_{f} \simeq \frac{e E_{\mathrm{SP}} \gamma_{p}^{2}}{k} \simeq m_{e} c^{2} a_{\mathrm{SP}} \alpha, \quad \tan \phi_{e} \equiv \frac{p_{x}}{p_{y}} \simeq \gamma_{p}^{-1}$.

Thus, strongly relativistic electrons $\left(\mathcal{E}_{f} \gg m_{e} c^{2}\right)$ are emitted at small $\phi_{e}$, i.e., close to the target surface. The acceleration length $\ell_{a} \equiv \mathcal{E}_{f} / e E_{\mathrm{SP}} \simeq \lambda \alpha / 2 \pi$, showing that electrons may reach the highest energy over a few microns. For linear conversion of the laser pulse into the surface plasmon, $a_{\mathrm{SP}} \propto a_{0}$ is expected. Notice, however, that for high amplitudes $a_{\mathrm{SP}}$ may be limited by wave breaking effects.

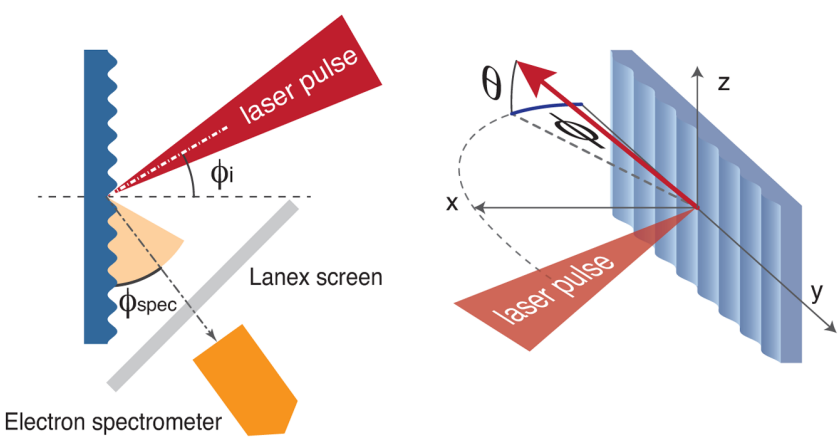

FIG. 1. Schematic view of the experimental setup. The 2D top view (left) shows the position of the diagnostics. The 3D sketch (right) shows the adopted convention for the angles $\phi$ and $\theta$.

The experiment was carried out at the CEA Saclay Laser Interaction Center (SLIC) facility with the UHI100 laser system (see Ref. [24] for a preliminary presentation of the experimental results). The laser pulse had $0.8 \mu \mathrm{m}$ wavelength, 25 fs duration, and $2.3 \mathrm{~J}$ energy before compression. A double plasma mirror [25] yielded a pulse contrast $\sim 10^{12}$ managing $\sim 50 \%$ energy loss. The pulse was focused with $P$ polarization using an off-axis $f / 3.75$ parabola in a focal spot of $\simeq 4 \mu \mathrm{m}$ FWHM containing $\sim 60 \%$ of the total energy in the $1 / e^{2}$ spot diameter, which lead to an average intensity of $\sim 5 \times 10^{19} \mathrm{~W} / \mathrm{cm}^{2}$. Focal spot optimization was performed with an adaptive optical system. The schematic view of the experimental setup is shown in Fig. 1. A compact CMOS-based spectrometer, specifically designed for this experiment, was mounted on a motorized tray able to change the angle $\phi_{\text {spec }}$ within the range $0^{\circ}-60^{\circ}$ from the tangent, while remaining aligned to the interaction center. The entrance lead pinhole had a diameter of $500 \mu \mathrm{m}$ and was placed at $8 \mathrm{~cm}$ from the interaction point. A pair of permanent magnets dispersed the electrons on the large $\left(49.2 \times 76.8 \mathrm{~mm}^{2}\right)$ triggered 12bit CMOS with $48 \mu \mathrm{m}$ pixel size. The energy detection range was $\sim 2-30 \mathrm{MeV}$. A scintillating Lanex screen $\left(16 \times 5 \mathrm{~cm}^{2}\right)$ was used to collect the electron spatial distribution in the angular range $\phi=0^{\circ}-90^{\circ}$. The screen was placed with an angle of $45^{\circ}$ with respect to the target and its center was at $8 \mathrm{~cm}$ from the interaction point. The green light emitted by the Lanex was selected using a $546 \mathrm{~nm}$ band-pass filter and recorded by a 12 bit CCD. When in use, the Lanex screen excluded the electron spectrometer. In addition to the electron diagnostics, a Thomson parabola was used to detect protons emitted along the rear target normal, as in a previous experiment [15]. The proton energy cutoff was used as a reference to optimize the target position.

The grating targets were produced at Czech Technical University, Prague by heat embossing of Mylar ${ }^{\mathrm{TM}}$ foils using a metallic master. Mylar was chosen considering its high damage threshold for prepulses. In the following we show results obtained with targets with a resonant angle 


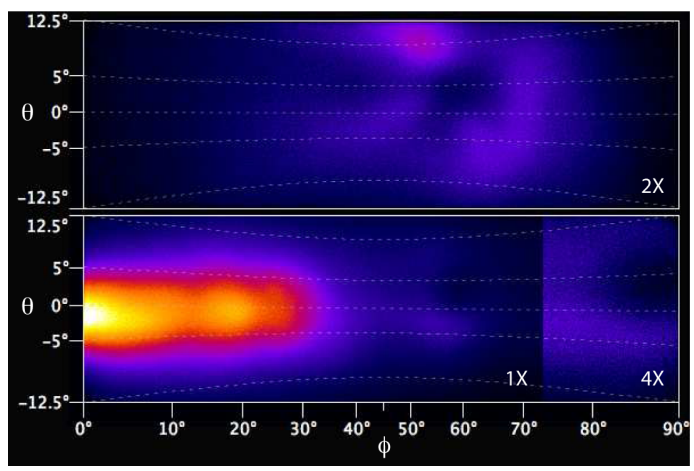

FIG. 2. Images on the Lanex screen for simple flat target (top) and for grating target (bottom), both irradiated at $\phi_{i}=30^{\circ}$ incidence. A $3 \mathrm{~mm}$ Al foil was placed in front of the screen to filter out electrons with energy $E<1.2 \mathrm{MeV}$. The parabolic dashed lines give the local $\theta$ angle corresponding to the position on the screen. In order to highlight the features of the signals, the upper panel has been amplified $2 \times$ compared to the bottom image. The right end $\left(\Phi>72^{\circ}\right)$ of the bottom image has been amplified too $(4 \times)$ to bring out the first-order diffraction minimum.

of $\simeq 30^{\circ}$, i.e., $\lambda_{g}=2 \lambda$ having assumed $\omega_{p} \gg \omega$. The average thickness was $10 \mu \mathrm{m}$ and the peak-to-valley depth of the grooves $0.25 \mu \mathrm{m}$. Flat foils with the same average thickness were used for comparison. In a limited number of shots, gratings with a resonance angle of $15^{\circ}\left(\lambda_{g}=1.35 \lambda\right)$ and $45^{\circ}\left(\lambda_{g}=3.41 \lambda\right)$ were also used, obtaining similar results.

Figure 2 shows the spatial distribution of the electrons for both gratings irradiated at angles near resonance and the flat foils. The emission from the flat foil is rather diffused, with a local minimum in correspondence of the specular reflection direction, as if electrons were swept away by the reflected pulse. The signal is maximum in an annular region around the hole. In contrast, for a grating at resonance the emission is strongly localized on the plane of incidence $\left(\theta \sim 0^{\circ}\right)$. The maximum intensity of the electron signal (in units of $1 / \mathrm{sr}$ ) is close to the target tangent and is $\sim 10$ times larger than the maximum intensity observed for flat targets at the same angle of incidence. Two minima ("holes" in the image) are observed in the directions of specular reflection and first-order diffraction of the laser pulse (evidence of grating diffraction of the high-intensity pulse, which confirms the survival of the grating during the interaction, was also found in previous measurements [15]). Local bending of the target or nonexact perpendicularity of the grating grooves to the plane of incidence may result in shot-to-shot fluctuations of the direction of maximum emission. Depending on the individual foil, the average angular shift in $\theta$ was in the $1^{\circ}-5^{\circ}$ range. An optimization of target design and alignment is foreseen to eliminate the fluctuations. The amount of charge accelerated in the collimated bunch along the target surface was estimated from the absolute calibration of the Lanex screen, resulting on

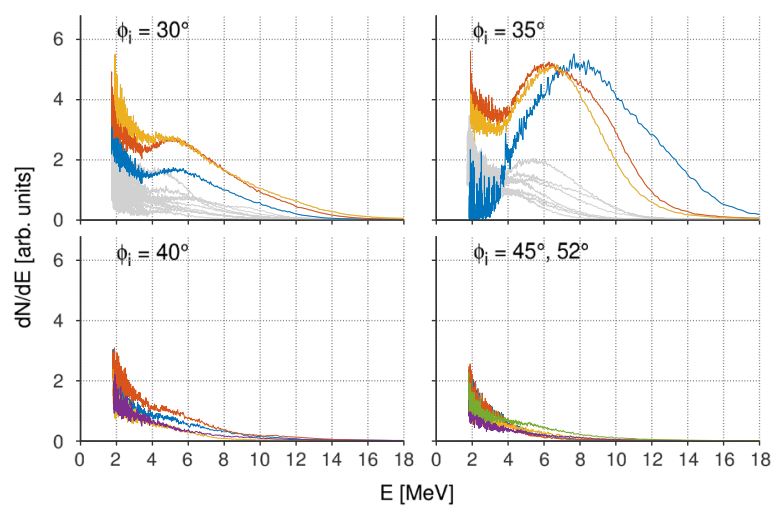

FIG. 3. Electron spectra collected with the detector at $2^{\circ}$ from tangent direction, for several pulse incidence angles (from $30^{\circ}$ to $52^{\circ}$ ). In the upper panels, all the collected shots are shown and a few of them are highlighted in color, while the others are plotted in light grey to show the shot-to-shot variations.

average in $100 \pm 15 \mathrm{pC}$, spread in a $8.5^{\circ}$ full angle cone. As a comparison, the overall emission from the flat foil around the specular reflection $(m=0)$ was estimated in a square region $\left(35^{\circ}\right.$ full angle in $\phi$ and $\theta$ ), resulting in about $60 \mathrm{pC}$.

The energy spectra were obtained placing the spectrometer at $\phi_{\mathrm{spec}}=2^{\circ}$. The angle of incidence $\phi_{i}$ was varied from $20^{\circ}$ to $52^{\circ}$. Figure 3 shows spectra obtained for $\phi_{i} \geq 30^{\circ}$, as for smaller angles no signal above the noise level was collected. The aforementioned fluctuations of the direction of the electron beam lead to a shot-to-shot variability of the intensity of the signal. Nevertheless, the most intense signals are detected only close to the resonance angle $\left(\sim 30^{\circ}\right)$. Moreover, spectra collected at $30^{\circ}$ and $35^{\circ}$ are characterized by higher maximum energies and a peculiar distribution with a dip at lower energies (3-4 MeV) and a broad peak at 5-8 MeV, and a highenergy tail extending up to $\sim 20 \mathrm{MeV}$. Electron spectra above the noise level from flat targets at $\phi_{\text {spec }} \sim 2^{\circ}$ were not observed for any incidence angle. Spectra obtained for $\phi_{i}=30^{\circ}$ and changing $\phi_{\text {spec }}$ in the $1^{\circ}-35^{\circ}$ range showed a similar shape in all positions of the spectrometer when $\phi_{\text {spec }} \lesssim 20^{\circ}$. The signal monotonically decreased in intensity with respect to $\phi_{\text {spec }}$ and was visible up to $\phi_{\text {spec }} \simeq 30^{\circ}$, in agreement with the signal collected on the Lanex screen.

3D simulations were performed for flat targets at $\phi_{i}=$ $30^{\circ}$ and for gratings at $\phi_{i}=30^{\circ}, 35^{\circ}, 40^{\circ}$. For computational feasibility, the target thickness was $\ell_{t}=1 \lambda$ and the electron density was $n_{e}=50 n_{c}$ (where $n_{c}=\pi m_{e} c^{2} / e^{2} \lambda^{2}$ is the cutoff density), while the other parameters corresponded to the experimental ones. The simulations were performed on 16384 cores of the FERMI supercomputer using the open-source, particle-in-cell code "PICCANTE" $[26,27]$. The numerical box size was $80 \lambda \times 80 \lambda \times 60 \lambda$, wide enough for the boundaries not to affect the results. A resolution of $70 \times 51 \times 34$ points per $\lambda$ and 50 particles per cell were used. 


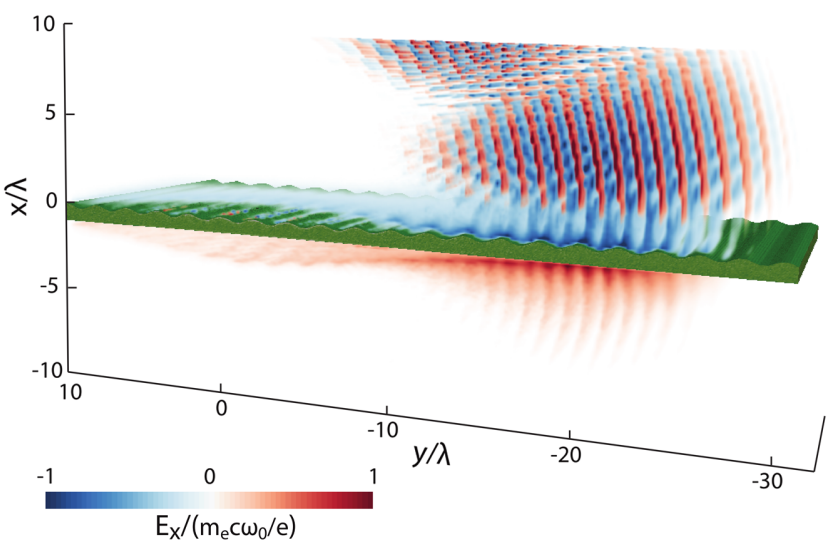

FIG. 4. 3D particle-in-cell simulation snapshot of laser-grating interaction, showing the electric field component $E_{x}$ and an isosurface highlighting the target at time $t=35 \lambda / c$ after the beginning of the interaction. Only the $z<0$ region of the simulation box is shown in order to highlight the field distribution. In the upper part of the picture, the distribution of $E_{x}$ is related to the diffracted pulse, while that in the lower part corresponds to a surface plasmon propagating in the $-\hat{y}$ direction.

Figure 4 shows a snapshot of a 3D simulation of a grating irradiated at the resonance angle in the same geometry of Fig. 1. The electric field component $E_{x}$ is represented together with the isosurface corresponding to the electron density. A surface plasmon propagating along the $-\hat{y}$ direction is excited. Figure 5(a) shows the simulated electron energy spectra $d N / d E$ at $\phi_{\text {spec }}=2^{\circ}$ for the flat target irradiated at $\phi_{i}=30^{\circ}$ and for gratings at $\phi_{i}=30^{\circ}, 35^{\circ}, 40^{\circ}$. With respect to the gratings, the signal for the flat target is much weaker and the energy cutoff is $\sim 10$ times lower. The spectrum for the grating at $\phi_{i}=30^{\circ}$ shows the peculiar shape observed for $\phi_{i}=30^{\circ}, 35^{\circ}$ in the experiment (Fig. 3), while for larger $\phi_{i}$ the low energy dip disappears, as for $\phi_{i} \geq 40^{\circ}$ in the experiment. Figure 5(b) compares the spectra obtained in 2D and 3D simulations, showing that details such as the broad peak with low energy dip are reproduced only in 3D. The angular distribution on the screen Fig. 5(c) also reproduces the experimental data (Fig. 2) including the hole in the specular reflection direction.

The 3D simulation also shows a correlation between electron energy and the emission angle. Electrons at energies lower than the peak value are emitted at some angle with respect to the propagation direction of the surface plasmon, so that the 3D spectrum integrated over the whole range of $\theta$ resembles the 2D case. This is consistent with interpreting the fluctuations in the energy spectra (Fig. 3) as related to those in the electron beam direction.

In the simulation, $a_{\mathrm{SP}} \simeq 1$ showing that the surface plasmon is relativistic. Inserting such a value and $\alpha=$ 50 in Eq. (2) we obtain a maximum energy $\mathcal{E}_{f} \simeq 25 \mathrm{MeV}$ and $\phi_{e} \simeq 8^{\circ}$, in fair agreement with the observations

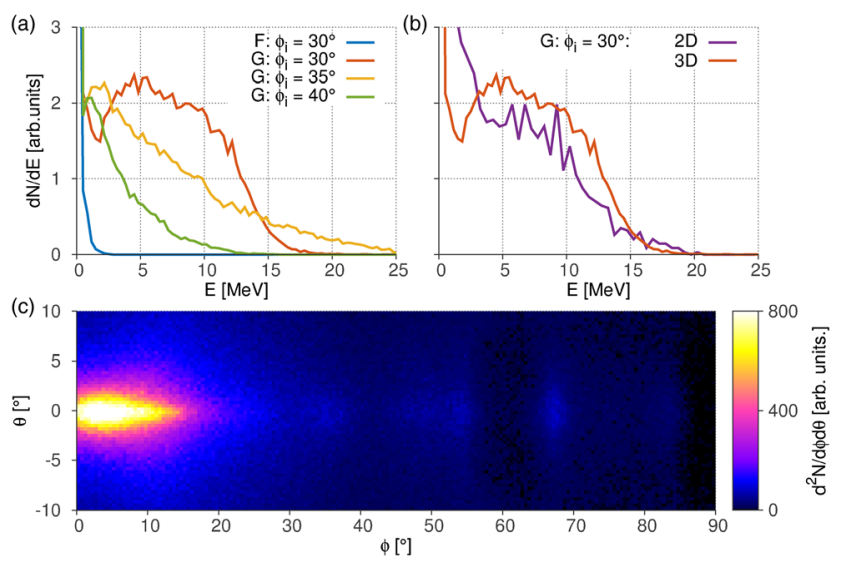

FIG. 5. Analysis of 3D simulations at time $t=45 \lambda / c$ after the beginning of the interaction. (a) Simulated spectrum $d N / d E$ at $\phi_{\text {spec }}=2^{\circ}$ for flat $(F)$ targets and for gratings $(G)$ at different values of the incidence angle $\phi_{i}$. Particles with $x<-0.125$ (the average surface position of the target), $|\phi-2|>0.5^{\circ}$ or $|\theta|>$ $0.5^{\circ}$ are filtered out. (b) Comparison of spectra between $2 \mathrm{D}$ and 3D simulation for a grating target irradiated at $\phi_{i}=30^{\circ}$. (c) Electron angular distribution $d^{2} N / d \phi d \theta$ for a grating irradiated at $30^{\circ}$. Particles with $x<-0.125$ or kinetic energy $E_{k}<1 \mathrm{MeV}$ are filtered out.

considering the simplicity of the model. 2D simulations show that $\mathcal{E}_{f} \propto a_{0}$ for $a_{0} \lesssim 10$ while the scaling is slower for higher $a_{0}$, presumably due to nonlinear effects. Details of the spectrum will be dependent on the distribution of injection velocities. The Supplemental Material [28] includes movies from 2D simulations showing the acceleration of electrons along the surface. In a very recent work [29], self-injection and phase locking of electrons in the surface plasmon wave is studied with a test-particle approach.

The proposed scheme, with further optimization, may provide an intense ultrashort electron source in the $\mathrm{MeV}$ range, with characteristics not easily attainable with other techniques and potential applications including photoneutron generation [30,31] or ultrafast electron diffraction [32-34]. The study of relativistic surface plasmons may be further pursued in various schemes inspired by ordinary plasmonics, such as tapered waveguides for energy concentration and field amplification [35,36], and exploiting various target structurings including optically controlled transient gratings [37]. Such development of high field plasmonics will open new possibilities for the control and manipulation of ultraintense laser pulses and their interaction with matter.

The research leading to these results has received funding from LASERLAB-EUROPE (Grant agreement No. 284464, EU FP7). Support from "Investissement d'Avenir" LabEx PALM (Grant ANR-10-LABX-0039), Triangle de la physique, Contract No. 2014-0601T ENTIER) and "Institut Lasers et Plasmas" is also acknowledged. Partial support of the Czech Science Foundation 
Project No. 15-02964S is gratefully acknowledged. We acknowledge ISCRA and LISA access schemes to the BlueGene/Q machine FERMI at CINECA, Italy, via the projects "FOAM2" (ISCRA) and "LAPLAST" (LISA), and PRACE for the development of the code within the project "PICCANTE." The Lanex screen calibration was performed at Laboratoire de l'Accélérateur Linéaire with the help of Thomas Vinatier, Pierre Lepercq, and Alexandre Gonnin. We thank the team of the Saclay Laser Interaction Center for their support, and Ondrej Klimo, Fabien Quéré, Gianluca Sarri, and Caterina Riconda for useful help and discussions.

*luca.fedeli@for.unipi.it

†Present address: Institute of Radiation Physics, HelmholtzZentrum Dresden-Rossendorf, 01328 Dresden, Germany.

[1] J. M. Pitarke, V. M. Silkin, E. V. Chulkov, and P. M. Echenique, Theory of surface plasmons and surfaceplasmon polaritons, Rep. Prog. Phys. 70, 1 (2007).

[2] S. A. Maier, Plasmonics: Fundamentals and Applications (Springer Science \& Business Media, New York, 2007).

[3] Surface plasmon resurrection (editorial), Nat. Photonics 6, 707 (2012).

[4] D. K. Gramotnev and S. I. Bozhevolnyi, Plasmonics beyond the diffraction limit, Nat. Photonics 4, 83 (2010).

[5] A. G. Brolo, Plasmonics for future biosensors, Nat. Photonics 6, 709 (2012).

[6] E. Ozbay, Plasmonics: Merging photonics and electronics at nanoscale dimensions, Science 311, 189 (2006).

[7] K. Quinn et al., Laser-Driven Ultrafast Field Propagation on Solid Surfaces, Phys. Rev. Lett. 102, 194801 (2009).

[8] S. Tokita, S. Sakabe, T. Nagashima, M. Hashida, and S. Inoue, Strong subterahertz surface waves generated on a metal wire by high-intensity laser pulses, Sci. Rep. 5, 8268 (2015).

[9] A. Gopal, S. Herzer, A. Schmidt, P. Singh, A. Reinhard, W. Ziegler, D. Brömmel, A. Karmakar, P. Gibbon, U. Dillner, T. May, H.-G. Meyer, and G. G. Paulus, Observation of Gigawatt-Class THz Pulses from a Compact Laser-Driven Particle Accelerator, Phys. Rev. Lett. 111, 074802 (2013); A. Poyé, S. Hulin, M. Bailly-Grandvaux, J.-L. Dubois, J. Ribolzi, D. Raffestin, M. Bardon, F. Lubrano-Lavaderci, E. D'Humières, J. J. Santos, Ph. Nicolaï, and V. Tikhonchuk, Physics of giant electromagnetic pulse generation in shortpulse laser experiments, Phys. Rev. E 91, 043106 (2015).

[10] G. Hu, A. Lei, W. Wang, X. Wang, L. Huang, J. Wang, Y. Xu, J. Liu, W. Yu, B. Shen, R. Li, and Z. Xu, Collimated hot electron jets generated from subwavelength grating targets irradiated by intense short-pulse laser, Phys. Plasmas 17, 033109 (2010); G. Hu, A. Lei, J. Wang, L. Huang, W. Wang, X. Wang, Y. Xu, B. Shen, J. Liu, W. Yu, R. Li, and Z. $\mathrm{Xu}$, Enhanced surface acceleration of fast electrons by using subwavelength grating targets, Phys. Plasmas 17, 083102 (2010); S. Bagchi, P. Prem Kiran, W.-M. Wang, Z. M. Sheng, M. K. Bhuyan, M. Krishnamurthy, and G. Ravindra Kumar, Surface-plasmon-enhanced $\mathrm{MeV}$ ions from femtosecond laser irradiated, periodically modulated surfaces, Phys. Plasmas 19, 030703 (2012).
[11] B. Dromey, S. Kar, M. Zepf, and P. Foster, The plasma mirror-a subpicosecond optical switch for ultrahigh power lasers, Rev. Sci. Instrum. 75, 645 (2004).

[12] C. Thaury, F. Quéré, J.-P. Geindre, A. Levy, T. Ceccotti, P. Monot, M. Bougeard, F. Reau, P. d'Oliveira, P. Audebert, R. Marjoribanks, and $\mathrm{Ph}$. Martin, Plasma mirrors for ultrahighintensity optics, Nat. Phys. 6, 424 (2008).

[13] M. Cerchez, A. L. Giesecke, C. Peth, M. Toncian, B. Albertazzi, J. Fuchs, O. Willi, and T. Toncian, Generation of Laser-Driven Higher Harmonics from Grating Targets, Phys. Rev. Lett. 110, 065003 (2013).

[14] M. A. Purvis, V. N. Shlyaptsev, R. Hollinger, C. Bargsten, A. Pukhov, A. Prieto, Y. Wang, B. M. Luther, L. Yin, S. Wang, and J. J. Rocca, Relativistic plasma nanophotonics for ultrahigh energy density physics, Nat. Photonics 7, 796 (2013).

[15] T. Ceccotti et al., Evidence of Resonant Surface-Wave Excitation in the Relativistic Regime through Measurements of Proton Acceleration from Grating Targets, Phys. Rev. Lett. 111, 185001 (2013).

[16] A. Macchi, F. Cornolti, F. Pegoraro, T. V. Liseikina, H. Ruhl, and V. A. Vshivkov, Surface Oscillations in Overdense Plasmas Irradiated by Ultrashort Laser Pulses, Phys. Rev. Lett. 87, 205004 (2001).

[17] A. Bigongiari, M. Raynaud, C. Riconda, and A. Héron, Improved ion acceleration via laser surface plasma waves excitation, Phys. Plasmas 20, 052701 (2013).

[18] M. Raynaud, J. Kupersztych, C. Riconda, J. C. Adam, and A. Héron, Strongly enhanced laser absorption and electron acceleration via resonant excitation of surface plasma waves, Phys. Plasmas 14, 092702 (2007).

[19] D. an der Brügge, N. Kumar, A. Pukhov, and C. Rödel, Influence of Surface Waves on Plasma High-Order Harmonic Generation, Phys. Rev. Lett. 108, 125002 (2012).

[20] K. Q. Pan, C. Y. Zheng, D. Wu, L. H. Cao, Z. J. Liu, and X. T. He, Study of strong enhancement of synchrotron radiation via surface plasma waves excitation by particlein-cell simulations, Appl. Phys. Lett. 107, 183902 (2015).

[21] P. K. Kaw and J. B. McBride, Surface waves on a plasma halfspace, Phys. Fluids 13, 1784 (1970).

[22] C. S. Liu, V. K. Tripathi, Xi Shao, and T. C. Liu, Nonlinear surface plasma wave induced target normal sheath acceleration of protons, Phys. Plasmas 22, 023105 (2015).

[23] T. Tajima and J. M. Dawson, Laser Electron Accelerator, Phys. Rev. Lett. 43, 267 (1979).

[24] L. Fedeli, A. Sgattoni, G. Cantono, I. Prencipe, M. Passoni, O. Klimo, J. Proska, A. Macchi, and T. Ceccotti, Enhanced electron acceleration via ultraintense laser interaction with structured targets, Proc. SPIE Int. Soc. Opt. Eng. 9514, 95140R (2015).

[25] A. Levy, T. Ceccotti, P. D’Oliveira, F. Réau, M. Perdrix, F. Quéré, P. Monot, M. Bougeard, H. Lagadec, P. Martin, P. Geindre, and P. Audebert, Double plasma mirror for ultrahigh temporal contrast ultraintense laser pulses, Opt. Lett. 32, 310 (2007).

[26] A. Sgattoni, L. Fedeli, and S. Sinigardi, Piccante: An OpenSource, Fully Relativistic, Massively Parallel Particlein-Cell Code, http://aladyn.github.io/piccante/.

[27] A. Sgattoni, L. Fedeli, S. Sinigardi, A. Marocchino, A. Macchi, V. Weinberg, and A. Karmakar, Optimising 
PICCANTE_an open source particle-in-cell code for advanced simulations on Tier-0 systems, arXiv:1503.02464.

[28] See Supplemental Material at http://link.aps.org/ supplemental/10.1103/PhysRevLett.116.015001 for animations showing the motion of some selected electrons in the fields near the grating surface. The color of the particles changes with the energy, showing that the strongest acceleration occurs along the surface. Also an animation showing the temporal evolution of the electric field in 3D is provided.

[29] C. Riconda, M. Raynaud, T. Vialis, and M. Grech, Simple scalings for various regimes of electron acceleration in surface plasma waves, Phys. Plasmas 22, 073103 (2015).

[30] H. Schwoerer, P. Gibbon, S. Düsterer, R. Behrens, C. Ziener, C. Reich, and R. Sauerbrey, MeV X Rays and Photoneutrons from Femtosecond Laser-Produced Plasmas, Phys. Rev. Lett. 86, 2317 (2001).

[31] Y. Arikawa et al., High-intensity neutron generation via laser-driven photonuclear reaction, Plasma Fusion Res. 10, 2404003 (2015).

[32] G. Sciaini and R. J. D. Miller, Femtosecond electron diffraction: heralding the era of atomically resolved dynamics, Rep. Prog. Phys. 74, 096101 (2011).
[33] S. Tokita, S. Inoue, S. Masuno, M. Hashida, and S. Sakabe, Single-shot ultrafast electron diffraction with a laser-accelerated sub-MeV electron pulse, Appl. Phys. Lett. 95, 111911 (2009).

[34] J. B. Hastings, F. M. Rudakov, D. H. Dowell, J. F. Schmerge, J. D. Cardoza, J. M. Castro, S. M. Gierman, H. Loos, and P. M. Weber, Ultrafast time-resolved electron diffraction with megavolt electron beams, Appl. Phys. Lett. 89, 184109 (2006).

[35] M. Esmann, S. F. Becker, B. B. da Cunha, J. H. Brauer, R. Ralf Vogelgesang, P. Groß, and C. Lienau, k-space imaging of the eigenmodes of sharp gold tapers for scanning nearfield optical microscopy, Beilstein J. Nanotechnol. 4, 603 (2013).

[36] M. I. Stockman, Nanofocusing of Optical Energy in Tapered Plasmonic Waveguides, Phys. Rev. Lett. 93, 137404 (2004).

[37] S. Monchocé, S. Kahaly, A. Leblanc, L. Videau, P. Combis, F. Réau, D. Garzella, P. D'Oliveira, Ph. Martin, and F. Quéré, Optically Controlled Solid-Density Transient Plasma Gratings, Phys. Rev. Lett. 112, 145008 (2014). 